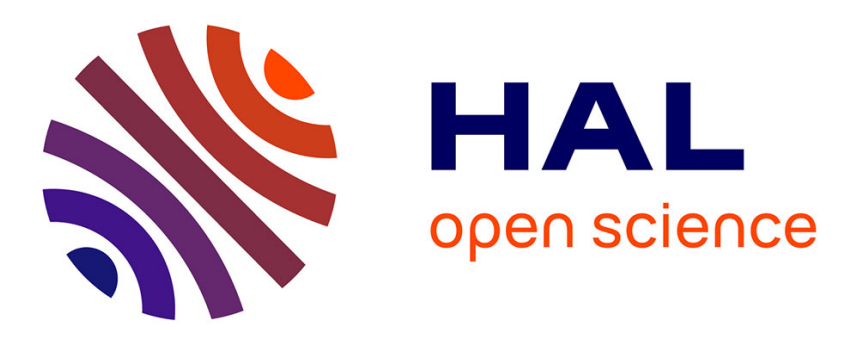

\title{
Pedestal formation of all-semiconductor gratings through GaSb oxidation for mid-IR plasmonics
}

Mario Bomers, Franziska Barho, Maria Jose Milla-Rodrigo, Laurent Cerutti, Richard Arinero, Fernando Gonzalez-Posada Flores, Eric Tournié, Thierry Taliercio

\section{To cite this version:}

Mario Bomers, Franziska Barho, Maria Jose Milla-Rodrigo, Laurent Cerutti, Richard Arinero, et al.. Pedestal formation of all-semiconductor gratings through GaSb oxidation for mid-IR plasmonics. Journal of Physics D: Applied Physics, 2018, 51 (1), pp.015104. 10.1088/1361-6463/aa98af . hal01754720

\section{HAL Id: hal-01754720 \\ https://hal.science/hal-01754720}

Submitted on 29 Mar 2019

HAL is a multi-disciplinary open access archive for the deposit and dissemination of scientific research documents, whether they are published or not. The documents may come from teaching and research institutions in France or abroad, or from public or private research centers.
L'archive ouverte pluridisciplinaire HAL, est destinée au dépôt et à la diffusion de documents scientifiques de niveau recherche, publiés ou non, émanant des établissements d'enseignement et de recherche français ou étrangers, des laboratoires publics ou privés. 


\title{
Pedestal formation of all-semiconductor gratings through GaSb oxidation for mid-IR plasmonics
}

\author{
Mario Bomers, ${ }^{1,2,}{ }^{*}$ Franziska Barho, ${ }^{1,2}$ María José Milla-Rodrigo, ${ }^{1,2}$ Laurent Cerutti, ${ }^{1,2}$ Richard Arinero, ${ }^{1,2}$ \\ Fernando Gonzalez-Posada Flores, ${ }^{1,2}$ Eric Tournié, ${ }^{1,2}$ and Thierry Taliercio ${ }^{1,2}$ \\ ${ }^{1}$ Université de Montpellier, IES, UMR 5214, F-34000, Montpellier, France \\ ${ }^{2}$ CNRS, IES, UMR 5214, F-34000, Montpellier, France \\ *mario.bomers@umontpellier.fr
}

\begin{abstract}
Mid-IR localized surface plasmon resonances (LSPR) have been demonstrated in nano-ribbons of highly Si-doped InAsSb alloys on GaSb substrates. We show that the slow, steady and selective oxidation of GaSb in water leads to an all-semiconductor mid-IR pedestal configuration consisting of highly doped InAsSb plasmonic resonators on top of $\mathrm{GaSb}$ pedestals embedded in an amorphous oxide layer. The homogeneity of the pedestal structure is imaged with an attenuated-total reflection Fourier transform infrared (ATR-FTIR) microscope by measuring around the plasmonic excitation at the plasma wavelength of $5.5 \mu \mathrm{m}$. As the plasmonic properties are influenced by modifications of the surrounding medium, we show for all-semiconductor gratings with defined doping level and defined grating geometry, that the GaSb oxidation process allows post-fabrication targeting of mid-IR plasmonic resonances to cover the mid-IR range from 5 to $20 \mu \mathrm{m}$. Additionally the pedestal formation reduces the refractive index mismatch between the two interfaces of the plasmonic resonators which allows to exploit a second plasmonic peak and which favors plasmonic field enhancement at the top (air-) side of the structure relevant for enhanced molecular vibration spectroscopy.
\end{abstract}

\section{Introduction}

The mid-IR range from 5 to $20 \mu \mathrm{m}$ is rich in molecular spectral signatures which enable the identification and quantification of unknown substances. The promise of plasmonics is on the one hand the potential to downscale molecular detection devices by exploiting field enhancement due to light concentration below the diffraction limit for surface enhanced infrared spectroscopy [1-7] and on the other hand the possibility to selectively absorb light for mid-IR detectors [8]. Research in the scientific field of mid-IR plasmonics stands on the shoulder of those who have pioneered with metal systems. The abundance of free charge carriers make metals very suitable for plasmonic application in the visible range of light, but limit their application in the mid-IR range due to increasing damping losses with increasing wavelength [9]. A recent review [10] discusses different plasmonic materials for the mid-IR range. In particular, the InAs material is unique for being a low-loss highly-doped semiconductor (HDSC) with the possibility to cover a tunable range of plasma wavelength down to $5 \mu \mathrm{m}$.

From a technological point of view, it is beneficial to work with the derivative $\operatorname{InAs}_{0.9} \mathrm{Sb}_{0.1}$ as it can be grown lattice matched on GaSb substrates and the electrons remain confined due to the large conduction band offset [11]. This property allows integrating mid-IR plasmonic features into compound devices like InAs/GaSb super-lattice photodetectors [12]. Another advantage is that the combination of $\mathrm{GaSb}$ and InAsSb allows selective etching for nanofabrication [13].

While a process to modify the supporting layer of gold nanostructures to improve IR-sensing applications was reported recently [14], it remained to show that a similar process can be achieved by an all-semiconductor approach. Instead of creating a pedestal by removing parts of the high-refractive index substrate by etching methods $[14,15]$, or by bottom-up growth techniques [16], we create a pedestal configuration by a local material transition from crystalline $\mathrm{GaSb}$ to amorphous oxide. The resulting pedestal configuration allows to blue-shift the main localized surface plasmon resonance (LSPR) and the exploitation of a second plasmonic peak. We show and discuss that the fabricated all-semiconductor pedestal structure might find application in the field of surface enhanced infrared absorption spectroscopy (SEIRA), because additionally to the established advantages of (1) CMOS-compatibility and (2) doping-control to target mid-IR resonances, we can demonstrate a successful (3) pedestal-formation for resonance-shifting, for enhancement of a second plasmonic peak and for resonance shape-modification with favors plasmonic field enhancement at the top (air-) side of the structure relevant for molecular sensing.

To obtain pedestals for an all-semiconductor grating structure, we apply the slow, steady and selective oxidation of $\mathrm{GaSb}$ in $\mathrm{H}_{2} \mathrm{O}$ to modify the refractive index of the environment of plasmonic $\mathrm{InAs}_{0.9} \mathrm{Sb}_{0.1}$ grating structures. Many 
studies were dedicated to the thermal, anodic and plasma oxidation of III-V semiconductor [17]. Early works on anodic oxidation of GaSb [18,19] and on native oxide films [20] were pursued [21] and till now the oxidation process of $\mathrm{GaSb}$ is studied experimentally [22] and theoretically [23]. In a recent work, the role of $\mathrm{H}_{2} \mathrm{O}_{2}$ and $\mathrm{H}_{2} \mathrm{O}$ in $\mathrm{GaSb}$ reduction-oxidation reactions was investigated in detail [24]. The authors concluded that contrary to other III$\mathrm{V}$ material $\mathrm{GaSb}$ is oxidized by $\mathrm{H}_{2} \mathrm{O}$ and that without an additional etchant $\mathrm{H}_{2} \mathrm{O}_{2}$ can inhibit oxidation despite being a strong oxidizer for other III-V materials. In short, while InAs is stable in $\mathrm{H}_{2} \mathrm{O}$ [25], GaSb oxidizes in water. Several studies mention the oxidation process in water, but most focus on the first hours after immersion [26]. In this work we investigate the oxidation of GaSb up to 81 hours of immersion in water and we map the homogeneity of this process with an attenuated total reflection microscope.

\section{Sample fabrication and experimental setup}

The structure used in this study was grown on a Te-doped (001) GaSb substrate by solid-source molecular beam epitaxy (MBE). After thermal de-oxidation, a $300 \mathrm{~nm}$ thick non-intentionally doped GaSb buffer was grown, followed by $100 \mathrm{~nm}$ thick Si-doped $\mathrm{InAs}_{0.9} \mathrm{Sb}_{0.1}$. A doping level of $5 \times 10^{19} \mathrm{~cm}^{-3}$ corresponding to a plasma wavelength of $5.5 \mu \mathrm{m}$ was determined for the InAs $\mathrm{As}_{0.9} \mathrm{Sb}_{0.1}$ layer [27].The gratings were fabricated by UV-lithography and selective etching with $\mathrm{C}_{6} \mathrm{H}_{8} \mathrm{O}_{7}: \mathrm{H}_{2} \mathrm{O}_{2}$ as described in a previous work [28].

The water used for GaSb oxidation was purified by the Purelab Option-Q and had a resistivity of $13.4 \mathrm{M} \Omega \cdot \mathrm{cm}$ at $23^{\circ} \mathrm{C}$. Before immersion into water, the grating geometry was characterized with a Fei Inspect S-50 scanning electron microscope (SEM) at an electron-beam energy of $20 \mathrm{kV}$. Additionally, the gratings were measured with a Nanoscope IV atomic force microcope (AFM) with a cantilever (PPP-NCH-10, Nanosensors). The gratings were optically investigated with a Hyperion 3000 IR microscope coupled to a Vertex 70 Fourier-transform IR (FTIR) spectrometer. The light originating from a globar source was focused onto the sample with a x15 magnification Cassegrain objective $(\mathrm{NA}=0.4)$ to an imaging area of $100 \times 100 \mu \mathrm{m}^{2}$, respectively to an aperture reduced image area of $50 \times 50 \mu \mathrm{m}^{2}$. A polarizer in the beam path enabled polarization dependent detection of the reflection signal with a single element mercury cadmium telluride detector. As the commercially available GaSb substrate was Te-doped and thus partly absorptive in the investigated frequency range, from 500 to $4000 \mathrm{~cm}^{-1}$, the transmittance of the structure could not be measured. Additional to the single element detector, the focal plane detector (FPA) of the Hyperion 3000 IR microscope in combination with its attenuated total reflection (ATR) module was used for measurements. The 4096 pixel (64x64) FPA is sensitive between $800-4000 \mathrm{~cm}^{-1}$ and the ATR module consists of an x20 objective which couples incident light with angles from $10^{\circ}-24^{\circ}$ into a Ge-waveguide crystal.

\section{Reflectance measurements and discussion}

Two gratings with different ribbon width, but with the same grating period, the same height and made from the same MBE-grown wafer were investigated in this study. After pre-characterization, the grating structures were put into a water-filled beaker with the backside GaSb wafer side in contact with the glass. Subsequently, the sample were dried by nitrogen gas flow and characterized by FTIR. The grating with the smaller ribbon width was additionally investigated by AFM after each immersion step. This measurement cycle was repeated to obtain variations in dependence of the immersion time in water. To obtain a reflectance spectrum, the measured reflectivity was normalized by the reflectivity of a gold mirror which is reflecting nearly $100 \%$ of the incident light in the relevant wavelength range. In Fig. 1(a), the reflectance of the grating with smaller ribbon width is shown for quasi-normal incident light polarized perpendicular to the ribbon (see inset for illustration). After each oxidation step, i.e. immersion in water and subsequent drying with nitrogen gas, the reflection was measured under ambient conditions. A strong plasmonic feature $\mathrm{M} 1 *$ can be seen around $730 \mathrm{~cm}^{-1}(13.7 \mu \mathrm{m})$ before we started the immersion cycles $(0 \mathrm{~h}$ immersion time). The oxidation provokes a blue-shift and a strong shape modification of this main plasmonic peak M1 and the strong enhancement of a second peak M2.

In Fig. 1(b), the reflectance of a second grating with larger ribbon width is shown (light polarized perpendicular to the ribbon). The geometrical dimensions could be obtained from SEM measurements (ribbon width $1050 \mathrm{~nm}$ $( \pm 50 \mathrm{~nm}$ ), grating period of $2 \mu \mathrm{m})$. For illustrative purposes we have added a SEM images of the grating before the oxidation process. Due to the larger ribbon width, the main resonance before the immersion is with $576 \mathrm{~cm}^{-1}$ $(17.4 \mu \mathrm{m})$ red-shifted compared to the grating with smaller ribbon width [28]. The general trend of blue-shifting, shape modification and the enhancement of a second peak upon GaSb oxidation is similar to the results obtained for the grating with smaller ribbon width presented in Fig. 1(a). The oxidation reaction is slower for the grating with larger ribbons as less of the GaSb surface is exposed to water during immersion. Fig. 1 shows that the oxidation process is repeatable and we focus the discussion in the following on the grating with smaller ribbon width. 

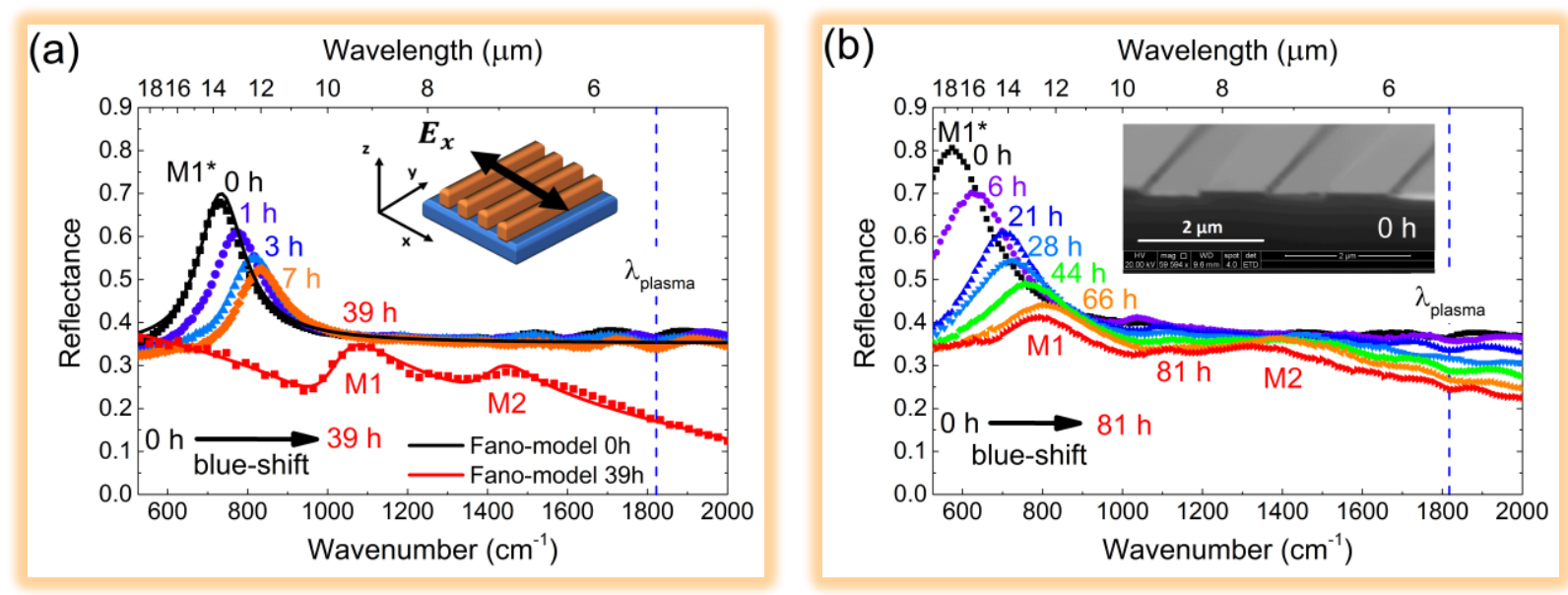

Fig. 1: Reflection measurement on $\mathrm{InAs}_{0.9} \mathrm{Sb}_{0.1}$ gratings upon oxidation of the GaSb substrate in water. The plasma wavelength of the highly-doped $\mathrm{Si}$-doped $\mathrm{InAs} \mathrm{A}_{0.9} \mathrm{Sb}_{0.1}$ is indicated. (a) The main reflection peak of a grating with smaller ribbon width blue-shift from $0 \mathrm{~h}$ immersion in water to finally $39 \mathrm{~h}$ immersion in water. The reflectance spectra before substrate oxidation and after $39 \mathrm{~h}$ of immersion in water (solid lines) are fitted by the Fano-model (Equation 2). Inset: Sketch of experimental configuration. $E_{x}$ represents the electric orientation of polarized-light. (b) The repeatability of the oxidation process, i.e. the resonance blue-shifting of a grating with larger ribbon width is shown. Inset: SEM images before immersion.

Qualitatively the blue-shift can be understood by considering the excitation condition for localized surface plasmons [7,29-31]. In the $x-y$-plane, see inset in Fig. 1(a), only $E_{x}$-polarization (perpendicular to the grating) allows to couple incident light to surface plasmons. The absence of plasmonic features in $\mathrm{E}_{\mathrm{y}}$-polarization (in direction of the gratings) underlines the LSPR argument (reflectance not shown). The simplest analytical formula for LSPR can be derived from Mie theory for spherical metallic particles with diameter smaller than the wavelength of incident light, where the resonance condition is $\varepsilon(\omega)=-2 \cdot \varepsilon_{\text {env }}$ [29]. Here, $\varepsilon_{\text {env }}$ and $\varepsilon(\omega)$ are the dielectric functions of the environment and of the HDSC. Upon oxidation $\varepsilon_{\mathrm{env}}$ is reduced and by considering the monotonicity of $\varepsilon(\omega)$, described by the Drude model, this leads to blue-shift the plasmonic features.

The change from symmetric to asymmetric line-shape of the main reflectance peak in Fig. 1 can be discussed in the framework of geometrical optics as done by Huck et al. [14] and in the framework of Fano-resonances [32,33] because both deal with phase shift and interferences. In the context of the Fano-model, the incident light is considered as a continuum of states (with broad band width) which interacts with the plasmonic nano-scale system of discrete states (with narrow band width). The resulting Fano-resonance can be described by

$$
F(\Omega)=\frac{(\Omega+q)^{2}}{\left(\Omega^{2}+1\right)},(1)
$$

with $\Omega=\left(\omega-\omega_{L S P R}\right) /\left(\gamma_{L S P R}\right)$, where $\omega_{L S P R}$ is the LSPR-frequency, $\gamma_{L S P R}$ the corresponding spectral width and $\mathrm{q}$ the Fano asymmetry parameter. In this work the Fano-model allows to reduce the complexity of interference phenomena occurring around the plasmonic InAsSb-ribbons induced by the refractive index modification upon oxidation. The approach corresponds therefore to an effective medium approach. We use the Fano-model to fit the measured reflectance by following formula

$$
R(\omega)=B_{0}+B_{1} \cdot \omega+\sum_{i} A_{i}\left(1-\frac{\left(\Omega_{i}+q_{i}\right)^{2}}{\left(\Omega_{i}{ }^{2}+1\right)}\right) .
$$

The parameter $B_{0}$ accounts for a constant background reflectance and $B_{1}$ for a linear background reflectance contribution in the shown spectral range of Fig. 1(a). $A_{i}$ represents the amplitude of the Fano-resonance, $q_{i}$ the resonance asymmetry. $\Omega_{i}$ determines the spectral position $\omega_{L S P R, i}$ and the resonance width $\gamma_{L S P R, i}$ as defined above.

We find that the reflection before oxidation can be fitted by 5 parameters, i.e. by one resonance and by one parameter for the constant background reflectance. After 39h immersed in water, we obtain a good fit of the measured reflectance by 10 parameters accounting for two resonances and a linear and a constant background contribution. The determined parameters are summarized in Table 1. 


\begin{tabular}{c|cccccccccc} 
& $\mathrm{B}_{0}$ & $\mathrm{~B}_{1}[\mathrm{~cm}]$ & $\mathrm{A}_{1}$ & $\omega_{L S P R, 1}\left[\mathrm{~cm}^{-1}\right]$ & $\gamma_{L S P R, 1}\left[\mathrm{~cm}^{-1}\right]$ & $q_{1}$ & $\mathrm{~A}_{2}$ & $\omega_{L S P R, 2}\left[\mathrm{~cm}^{-1}\right]$ & $\gamma_{L S P R, 2}\left[\mathrm{~cm}^{-1}\right]$ & $q_{2}$ \\
\hline $0 \mathrm{~h}$ & 0.35 & - & 0.35 & 730 & 70 & -0.05 & - & - & - & - \\
$39 \mathrm{~h}$ & 0.5 & -0.000195 & 0.07 & 1020 & 80 & -0.9 & 0.06 & 1380 & 80 & -0.5
\end{tabular}

Table 1: Parameters to fit the reflectance shown in Fig. 1(a) by Equation 2.

The fit parameters indicate that the magnitude of the asymmetry parameter $q$ increases significantly upon oxidation. While the magnitude is nearly zero before oxidation, $\mathrm{q}_{1}$ is close to one after oxidation. For the same allsemiconductor material system, another harmonization of the optical environment was already investigated. For HDSC ribbons embedded in a GaSb matrix [34] an asymmetry value near unity was found. We underline that increasing the matching of refractive index between the top-side and the supporting layer of the HDSC resonators increases the asymmetry parameter and causes a resonance shape modification. While $\mathrm{q}=0$ corresponds to resonant suppression of transmission, we find a reduction of this suppression through GaSb oxidation. We note that the asymmetry parameter for the second plasmonic resonance after $39 \mathrm{~h}$ of oxidation is in between zero and unity and we come back to this point in the FDTD section.

\section{Pedestal formation}

The reflectance measurements indicate clearly a modification of the optical properties of the plasmonic gratings upon oxidation. To investigate these change in more detail, the grating from Fig. 1(a) sample was cleaved and investigated by cross-section SEM after $39 \mathrm{~h}$ of immersion in water. Furthermore, we took an IR-image of the oxidized grating structure by using the FPA of the Hyperion 3000 IR microscope in combination with its ATR module.

We show in Fig. 2(a) a sketch of the pedestal formation by GaSb oxidation in water and in Fig. 2(b) a crosssection SEM image of the grating after $39 \mathrm{~h}$ immersion in water. A clear contrast originating from differently backscattered electrons allows distinguishing oxidized GaSb from non-oxidized GaSb. For the sake of illustration, we have chosen black to symbolize high backscattering and white to symbolize low backscattering. By energydispersive X-ray spectroscopy (EDS) the difference in the backscattered electron image can be attributed to different material composition. The two spectra shown in Fig. 2(c) present the electron excited X-rays detected from the indicated regions in Fig. 2(b). A database comparison allows to assign the peaks to core-electron transitions. The most significant feature is the increase of the peak at $0.53 \mathrm{keV}$. We attribute this increase in intensity to the incorporation of oxygen into the material. The increase in intensity of the Ga (L) peak and the decrease in intensity of the $\mathrm{Sb}(\mathrm{L})$ indicate a preferential dissolution of the group V-element, i.e. Antimony. For comparison, in the literature preferential dissolution of the group V-element by water was reported for GaAs [35]. 


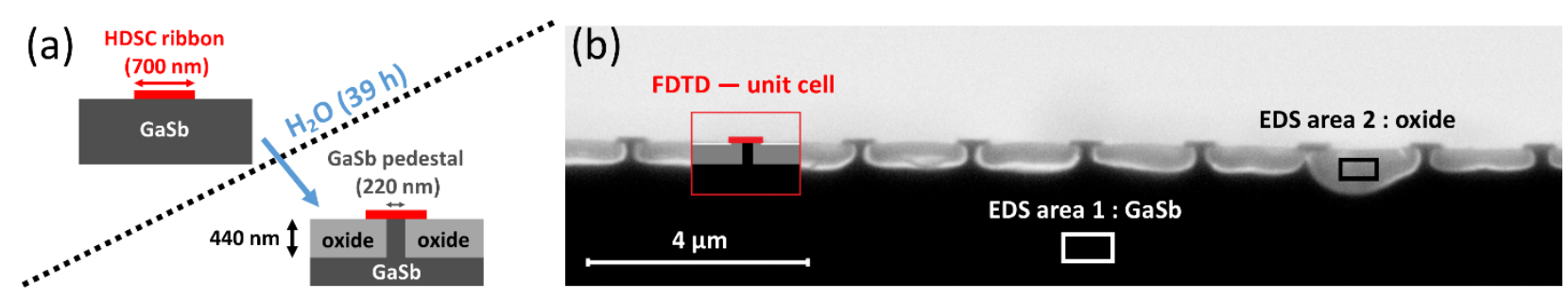

(c)

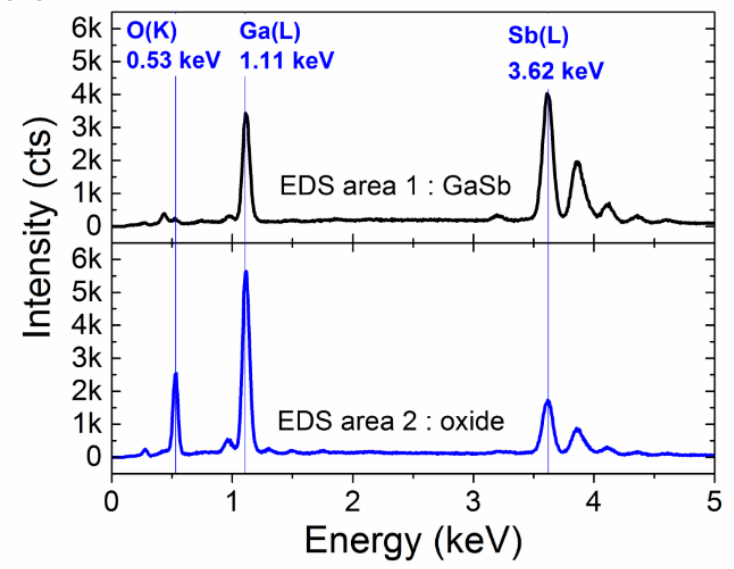

(d)

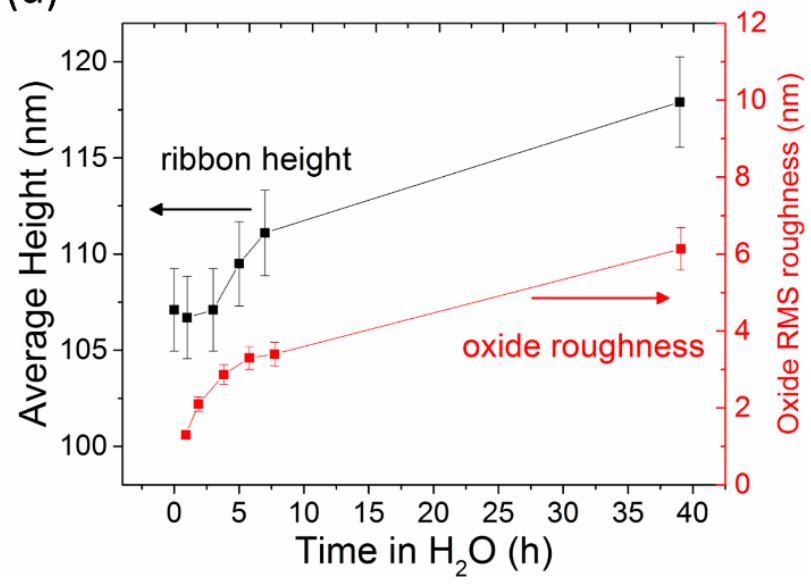

Fig. 2: (a) Sketch of the pedestal formation by GaSb oxidation in water. The dimensions of the FDTD-unit cell are shown. (b) Backscattering scanning electron microscopy (SEM) image of In $\mathrm{As}_{0.9} \mathrm{Sb}_{0.1}$ ribbons on partly oxidized GaSb layer (black = high backscattering, white $=$ low backscattering) after $39 \mathrm{~h}$ of immersion in water. (c) EDS spectra obtained in the areas indicated in the SEM image. (d) Average height of $\mathrm{InAs}_{0.9} \mathrm{Sb}_{0.1}$ gratings and root mean squared roughness value of oxide layer upon immersion time in water measured by atomic force microscopy.

By focusing with the SEM on the HDSC ribbons on top of the structure (not shown) we were able to determine the geometrical parameters of the InAs ${ }_{0.9} \mathrm{Sb}_{0.1}$ grating structure and the dimensions of the oxidized area. We used these parameters as input for a finite-difference time-domain model (see Fig. 2(a): FDTD unit cell). The AFM was used to determine the surface topography. In Fig. 2(d) the evolution of the surface topography is plotted over immersion time in $\mathrm{H}_{2} \mathrm{O}$. A statistical analysis of the area between the ribbons was performed to determine the root mean square (RMS) value of this area representative of the oxide roughness. The height of the ribbons was determined as average value of three ribbons. The error interval represents the measured deviations from this average value. It can be seen that the oxide roughness (RMS-value) and the average height of the grating increases with the immersion time. We attribute the increase of height of the nano-ribbons to a slow oxide dissolution process at a rate of $10 \mathrm{~nm}$ in $39 \mathrm{~h}(0.25 \mathrm{~nm} / \mathrm{h})$, which is significantly slower than the dissolution in electrolyte solutions. It was indeed found [21] that anodically grown GaSb oxide dissolves in an electrolyte solution of $\mathrm{pH} 6.1$ at a rate of $2.94 \mathrm{~nm} / \mathrm{h}$.

Combining the results from the SEM and the AFM measurements, the geometry of a grating unit cell before the oxidation process is described by following geometrical parameters: a $\operatorname{InAs}_{0.9} \mathrm{Sb}_{0.1} \mathrm{HDSC}$ ribbon with width of $700 \mathrm{~nm}( \pm 30 \mathrm{~nm})$, height of $107 \mathrm{~nm}( \pm 2 \mathrm{~nm})$ and unit cell length, i.e. grating period of $2000 \mathrm{~nm}( \pm 30 \mathrm{~nm})$. After the oxidation process ( $39 \mathrm{~h}$ immersion in water), an oxide layer with thickness of $440 \mathrm{~nm}( \pm 40 \mathrm{~nm})$ and RMSroughness of $6 \mathrm{~nm}$ was obtained. A small part of the oxide, $10 \mathrm{~nm}( \pm 2 \mathrm{~nm})$ was dissolved during the process. A pedestal of $\mathrm{GaSb}$ with a width of $220 \mathrm{~nm}( \pm 20 \mathrm{~nm})$ remained under the $\operatorname{InAs}_{0.9} \mathrm{Sb}_{0.1}$ ribbon. The oxidation is more pronounced vertically than horizontally as can be seen in Fig. 2(b). Additional to this anisotropy, the interfaces of the oxide and the GaSb pedestal appear curved in the SEM image which we neglect by describing the pedestal by one parameter, i.e. the pedestal width.

\section{FDTD modeling and discussion}

The geometrical parameters determined by SEM and AFM measurements were used as input for a finite-element model which allowed to solve Maxwell's equation by the finite difference time domain (FDTD) method using the commercial software FDTD-solution by Lumericals [36]. For GaSb, we chose a mid-IR refractive index value of $\mathrm{n}=3.77$ as reported recently [37]. The plasmonic properties of the highly-doped $\operatorname{InAs} \mathrm{As}_{0.9} \mathrm{Sb}_{0.1}$ can be described by the 
Drude model. Values of $\varepsilon_{\infty}=10.4, \omega_{\mathrm{p}}=1.11 \cdot 10^{15} \mathrm{rad} / \mathrm{s}$ and $\gamma=1 \cdot 10^{13} \mathrm{rad} / \mathrm{s}$ were obtained as fitting parameters [28]. Anodically grown native oxides were extensively studied in the visible and near-infrared range [19], but not for the mid-IR range of interest. We obtain good fitting of the measured reflectance spectra by assuming a value of $\mathrm{n}=1.6( \pm 0.1)$ for the oxide in the entire mid-IR range. The incidence angle due to the used objective is below $15^{\circ}$ and the wave vector contribution by not-normal light incident from air is negligible for the plasmonic subwavelength confinement in the given geometry. Therefore we have chosen normal incidence as input parameter for the FDTD simulations to decrease the calculation time.

The FDTD model allows to calculate the reflectance R and the absorption A of the nanostructure. In Fig. 3, the comparison of measured and simulated reflectance are in good agreement. The simulated reflectance for the oxidized sample is sharper than the measured one. We attribute this to the fact that our model idealizes the sharpness of boundaries. The SEM image (Fig. 2b) reveals variations of the oxide spreading and considering the $50 \times 50 \mu \mathrm{m}^{2}$ imaging area we expect broadening due to averaging effects. Details on the inhomogeneity effects will be discussed in the ATR-microscopy section.

With the FDTD model we could calculate the absorption of the plasmonic grating (see Fig. 3) and three plasmonic modes 1 $^{*}$, M1 and M2 can be distinguished. As the absorption A and the reflectance R, define the transmission $\mathrm{T}(=1-A-R)$ we find that only $\mathrm{M}^{*}$ correspond to resonant suppression of transmission and only $\mathrm{M} 1{ }^{*}$ has a symmetric reflection line-shape. For M1 and M2, the transmission is not resonantly suppressed and both have an asymmetric reflection line-shape. The difference in suppressed transmission between M1 and M2 explains the two different q-values for these resonances.

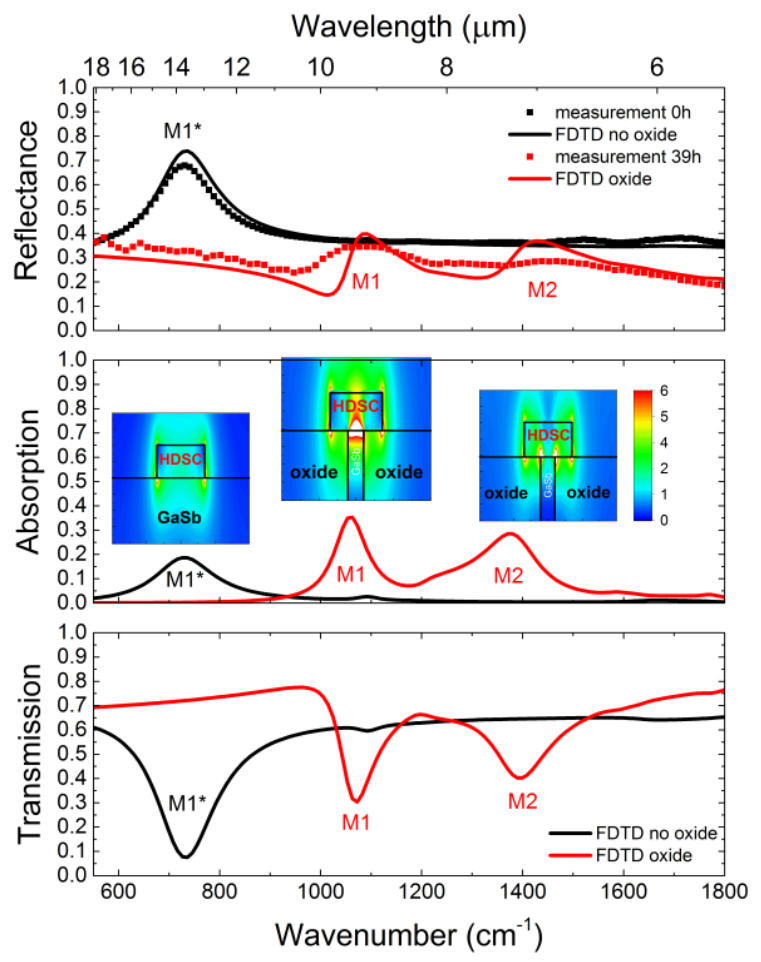

Fig. 3: Measured reflectance and FDTD calculated reflectance, absorption of HDSC grating and transmission into substrate. Near-field profile and measured reflectance before and after $39 \mathrm{~h}$ of immersion in water. The near-field images show the field-enhancement $\left|E / E_{0}\right|$ and were calculated at the respective absorption maxima of mode $\mathrm{M} 1 *, \mathrm{M} 1$ and $\mathrm{M} 2$. For the sake of better comparison, the color scale was limited to values from 0 to 6 in each image (white indicates an enhancement factor $>6$ ).

The main advantage of the FDTD model are the simulated near-field profiles of M1*, M1 and M2 at their respective maximum of absorption. These illustrate the influence of the oxidation and the influence of the remaining non-oxidized $\mathrm{GaSb}$-pedestal below the $\mathrm{InAs}_{0.9} \mathrm{Sb}_{0.1}$ nanostructure (Fig. 3). For M1* the E-field is mainly located at the two corners of the HDSC/GaSb interface. M1 is blue-shifted as large parts of the substrate are oxidized and only a GaSb pedestal remains. The main field-confinement of this mode is in the four corners of the HDSC ribbon and there is a strong confinement at the remaining HDSC/GaSb interface, why we will call it in the following the 
pedestal mode. The near-field profile of M2 is defined by E-field confinement in the four corners of the HDSC ribbon and at the HDSC/oxide interfaces. We will call this mode in the following the oxide mode.

In the pedestal configuration presented by Huck et. al. [14], which was fabricated by etching and consisted of three different optical materials (1) plasmonic material, (2) substrate material and (3) air, the height of the pedestal and the induced phase-shift by reflection at the air-substrate were the most relevant parameter. For our structure we have to consider the geometrical arrangement of four optically different material. The GaSb pedestal width and the oxide thickness are critical parameter. The advantage of working with four optically different material is the additional mode, i.e. having a pedestal mode (M1) and an oxide mode (M2).

An interesting observation is that the field-enhancement of the M1-mode and the M2-mode are more pronounced at the top (air-) side of the structure than for the M1* mode. This particularity might find application for surfaceenhanced infrared reflection spectroscopy (SEIRA), where the objective is to bring minute quantities of analyte material to the top-side of plasmonic resonators. The main conclusion from this part is that the oxide-pedestal interface of the HDSC is responsible for an additional plasmonic peak, that the plasmonic resonances blue-shift to target frequencies in dependence of the oxidation progress, i.e. the immersion time, and that the refractive index matching favors plasmonic field enhancement at the top (air-) side of the structure.

\section{Homogeneity mapping and discussion}

Attenuated total reflection Fourier-transform infrared (ATR-FTIR) microscopy was used to image the inhomogeneity of the oxidation process. As plasmonic features are strongly dependent on the oxide-pedestal interface, we address in this section the hypothesis that the difference between FDTD simulated reflectance and measured reflectance shown in Fig. 3 can be explained by resonance broadening due to averaging effects of inhomogeneous oxidation.

In Fig. 4(a) a sketch illustrates that the flat bottom side of the Hyperion $3000 \mathrm{Ge}$-crystal was brought into contact with the grating surface by exerting a pressure. The mid-IR refractive index of $\mathrm{Ge}\left(\mathrm{n}_{\mathrm{Ge}}=4\right)$ is comparable with the refractive index of $\mathrm{GaSb}\left(\mathrm{n}_{\mathrm{GaSb}}=3.77\right)$, but very different than the oxide value $\left(\mathrm{n}_{\text {oxide }}=1.6\right)$. The incident light from the globar source was coupled via an objective into the Ge-crystals top-side and the light was propagating through the crystal till the flat Ge-bottom interface in contact with the sample. The incident angle $\theta_{\text {in }}$ is in between $10^{\circ}$ and $24^{\circ}$. The major advantage of this experimental configuration is the increase of spatial resolution. The wavelength $\lambda$ dependent Rayleigh criterion of resolution equals $0.61 \lambda /\left(n \cdot \sin \left(\theta_{i n}\right)\right)$ and therefore we can spatially resolve the $2 \mu \mathrm{m}$ grating period down to the plasma wavelength of $5.5 \mu \mathrm{m}$ for light incident from the Germanium crystal $\left(n=n_{G e}\right)$.

The onset of total reflection is the critical angle $\left(14.5^{\circ}\right.$ for the Germanium-air interface). We used the spectra obtained in total reflection configuration for the Ge-crystal pending in air as a background spectra to normalize the reflection spectra measured in contact with the grating. As GaSb is like Ge a high-refractive index material, the critical angle for this interface is $70.5^{\circ}$ and most light incident through the Ge-crystal is transmitted into the GaSbsubstrate. Upon oxidation the refractive index decreases to 1.6 and the critical angle of the Germanium-oxide interface drops to $23.5^{\circ}$. For an incident angle of $24^{\circ}$ the light is totally reflected and an evanescent wave emerges at the interface. The evanescent wave penetrates into the underneath material at a typical depth

$$
d_{\text {pen }}=\frac{\lambda}{2 \pi n_{1} \sqrt{\left(\sin ^{2} \theta_{i n}-\left(n_{2} / n_{1}\right)^{2}\right)}}, \text { (3) }
$$

which corresponds to the electric field decreased to $\mathrm{e}^{-1}$ for a given incident angle $\theta_{\text {in }}$ and for given refractive index values of the top $n_{1}$ and bottom $n_{2}$ layer [38]. In the inset of Fig. 4(c), the value of $1 / d_{p e n}$ is plotted for the HDSC-Ge, air-Ge and the oxide-Ge interface in the mid-IR range for an incident angle of $24^{\circ}$. This explains that we have high reflectance in the near-IR, but low reflectance for higher wavelengths. In particular, the discussion of the critical angle points out that the substrate oxidation strongly increases the reflection signal and enables a much better signal to noise ratio compared to non-oxidized samples.

In Fig. 4(b), a FPA image is shown. The imaged area in the ATR-microscopy mode is $32 \times 32 \mu \mathrm{m}^{2}$ such that 1 pixel corresponds to a width of $500 \mathrm{~nm}$. The Rayleigh criterion requires a wavelength of $1.33 \mu \mathrm{m}$ to spatially resolve $500 \mathrm{~nm}$. The pixel resolution is sufficient to map the grating period of $2000 \mathrm{~nm}$ and the HDSC ribbon width of $700 \mathrm{~nm}$, but the angular resolution limits to fully exploit the pixel resolution. In particular for wavelength larger than the plasma wavelength of $5.5 \mu \mathrm{m}$ the diffraction broadening makes it difficult to address spatial inhomogeneity. The contrast of Fig. 4(b) is due to a numerical treatment of the measured reflectance. At each pixel the reflectance was integrated from 4.55 to $6.55 \mu \mathrm{m}$, i.e. around the plasma wavelength of the HDSC, and the background was removed by subtracting the area defined by the mean reflection and the integration boundaries. The reflectance within this integration boundaries varies by differently strong convex-curving as can be seen in Fig. 4(c). The color 
scale in Fig. 4(b) is such that red represents the strongest curving, blue represents lowest curving and green represents intermediate curving. It can be clearly seen that the contrast comes from the different reflectance for wavelength below the plasma wavelength. As diffraction broadening is less pronounced for these wavelength we can spatially resolve the ribbons.

In Fig. 4(c), the spectra obtained at the different pixels indicated in Fig. 4(b) are shown. From the near-IR to the plasma wavelength the ribbons are spatially still sufficiently resolved and more reflection signal is detected in between the ribbons than on the ribbons. We attribute this to the high refractive index of the HDSC in this wavelength range and to the remaining GaSb pedestal under the ribbons which leads to transmission into the $\mathrm{GaSb}$ substrate. Below the plasma wavelength, the HDSC becomes a low refractive index material and the inset of Fig. 4(c) illustrates that at an angle of incidence of $24^{\circ}$ an evanescent waves emerges. The integration area (upper and lower integration boundary) for the FPA-image are indicated in Fig. 4(c). It can be seen that due to the decreasing spatial resolution with increasing wavelength the contrast between spectra obtained at pixels associated with the ribbons and pixel in between the ribbons decreases.

To address the hypothesis of inhomogeneous oxidation and resonance broadening due to averaging, the scanning electron microscope top view image in the inset of Fig. 4(b) has to be compared with the FPA-image. While the electronic image shows a good homogeneity of the surface and the gratings, the optical image shows inhomogeneity. Within the $32 \times 32 \mu \mathrm{m}^{2}$ measurement area, we can see 16 ribbons (red) against the background (green). From the cross-section SEM image shown in Fig. 1(b), we know that inhomogeneous oxidation (different oxide height and different pedestal width) exist for some of the ribbons. We therefore interpret the disruption of the stripe pattern in the left corner of the FPA-image as such an inhomogeneous oxidation. Despite local variations, the spectra in Fig. 4(c) reveal that the overall reflection follows the trend of decreasing reflection with decreasing wavenumber. This shows that there are no zones without oxidation and just local variations. We conclude that the proposed FDTDmodel is sufficient to model the reflectance properties of the all-semiconductor mid-IR grating on pedestals, because the measured and simulated reflectance shown in Fig. 3 are in good agreement (obtained for a $50 \times 50 \mu \mathrm{m}^{2}$ measurement area with a single element detector). The deviations of FDTD model and measurement, in particular the resonance broadening, can be explained by an idealization of the boundaries in the model.
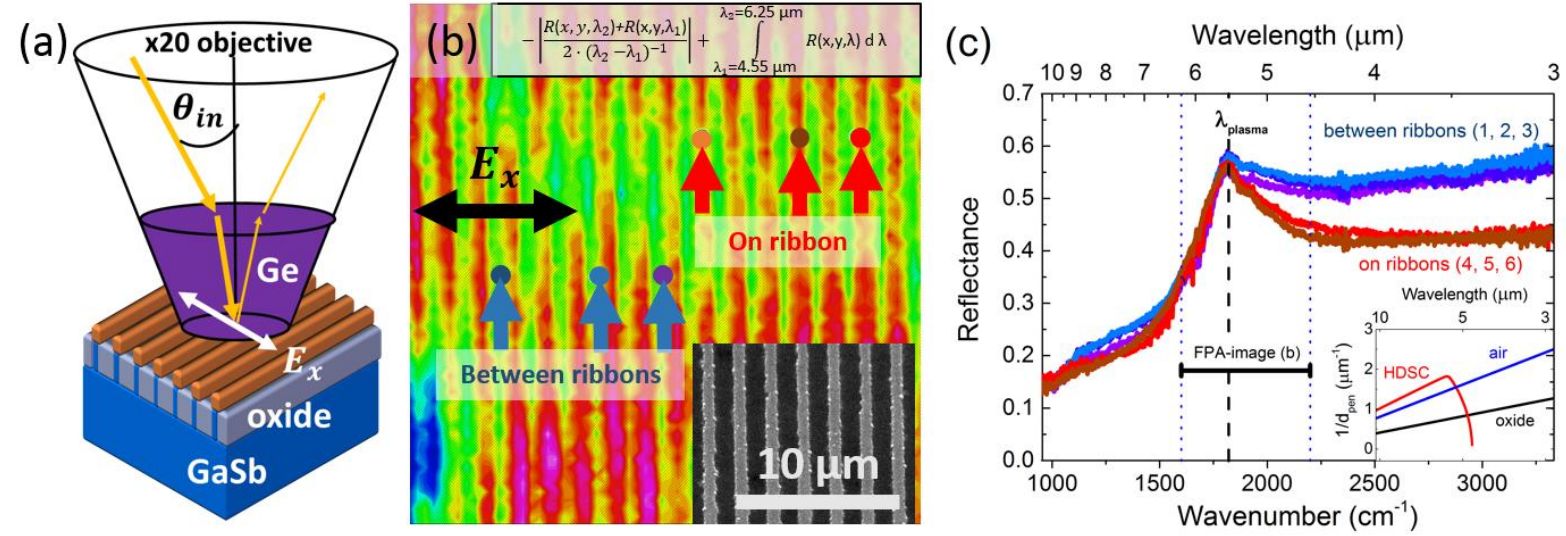

Fig. 4 : (a) Sketch of the experimental configuration. (b) Image of integrated and background subtracted reflectance spectra around plasma wavelength at $5.5 \mu \mathrm{m}$ obtained in ATR-configuration for x-polarized light. The inset shows a SEM image of the grating surface. (c) The spectra for the pixel indicated in (b) are shown. The calculated evanescent decay length is shown for an incident angle of $24^{\circ}$ (inset).

\section{Conclusion}

In this work we could show that the slow, steady and selective oxidation of $\mathrm{GaSb}$ in $\mathrm{H}_{2} \mathrm{O}$ allows to oxidize the supporting GaSb layer of a plasmonic InAs $\mathrm{Is}_{0.9} \mathrm{Sb}_{0.1}$ grating structures to create a pedestal configuration. The refractive index asymmetry of the plasmonic resonator's optical environment is reduced upon oxidation. This leads to a blueshifting of the main resonance and the enhancement of a second plasmonic resonance. The pedestal configuration additionally causes a stronger field-enhancement at the top (air-) side of the resonator. The homogeneity of the pedestal structure was imaged with an attenuated-total reflection Fourier transform infrared microscope by measuring around a plasmonic excitation at the plasma wavelength of $5.5 \mu \mathrm{m}$. We conclude that the chosen FDTDmodel is sufficient to explain the reflectance spectra. The measured local variations of the oxidation process lead to resonance broadening due to averaging effects which were not considered in our model. Finally, the controlled 
substrate oxidation is a post-fabrication technique to target plasmonic resonances in the mid-IR range from 5 to 20 $\mu \mathrm{m}$, to enhance a second plasmonic peak and to modify the near-field enhancement profile. This has great potential to find application in the field of surface-enhanced infrared reflection absorption spectroscopy.

Funding

French Investment for the Future program (EquipEx EXTRA, ANR 11-EQPX-0016); French ANR (SUPREME-B, ANR-14-CE26-0015); European Union's Horizon 2020 research and innovation programme (Marie SklodowskaCurie grant agreement No 641899); Occitanie region.

Acknowledgements

F. Pichot, J.-M. Peiris and J. Lyonnet are acknowledged for technical support at the cleanroom facilities of Université de Montpellier. G. Boissier, J.-M. Aniel and G. Narcy are acknowledged for technical support.

\section{References}

1. R. Aroca, Surface Enhanced Vibrational Spectroscopy (Wiley, 2006).

2. F. Neubrech, C. Huck, K. Weber, A. Pucci, and H. Giessen, "Surface-Enhanced Infrared Spectroscopy Using Resonant Nanoantennas," Chem. Rev. (2017).

3. F. B. Barho, F. Gonzalez-Posada, M.-J. Milla-Rodrigo, M. Bomers, L. Cerutti, and T. Taliercio, "Allsemiconductor plasmonic gratings for biosensing applications in the mid-infrared spectral range," Opt. Express 24, 16175 (2016).

4. M. J. Milla, F. Barho, F. González-Posada, L. Cerutti, B. Charlot, M. Bomers, F. Neubrech, E. Tournie, and T. Taliercio, "Surface-enhanced infrared absorption with Si-doped InAsSb/GaSb nanoantennas," Opt. Express 25, 26651-26661 (2017).

5. Thierry Taliercio, Fernando Gonzalez-Posada Flores, Franziska B. Barho, María José Milla-Rodrigo, Mario Bomers, Laurent Cerutti, and Eric Tournié, "Plasmonic bio-sensing based on highly doped semiconductors," in (2017), Vol. 10353, p. 103530S-10353-10.

6. R. R. Gutha, S. M. Sadeghi, and W. J. Wing, "Ultrahigh refractive index sensitivity and tunable polarization switching via infrared plasmonic lattice modes," Appl. Phys. Lett. 110, 153103 (2017).

7. R. R. Gutha, S. M. Sadeghi, C. Sharp, and W. J. Wing, "Biological sensing using hybridization phase of plasmonic resonances with photonic lattice modes in arrays of gold nanoantennas," Nanotechnology 28, 355504 (2017).

8. S. Ogawa, D. Fujisawa, H. Hata, M. Uetsuki, K. Misaki, and M. Kimata, "Mushroom plasmonic metamaterial infrared absorbers," Appl. Phys. Lett. 106, 041105 (2015).

9. A. Boltasseva and H. A. Atwater, "Low-Loss Plasmonic Metamaterials," Science 331, 290-291 (2011).

10. Y. Zhong, S. D. Malagari, T. Hamilton, and D. Wasserman, "Review of mid-infrared plasmonic materials," J. Nanophotonics 9, $093791-093791$ (2015).

11. T. Taliercio, V. Ntsame Guilengui, L. Cerutti, J.-B. Rodriguez, and E. Tournié, "GaSb-based allsemiconductor mid-IR plasmonics," in M. Razeghi, ed. (2013), p. 863120.

12. E. Giard, R. Taalat, M. Delmas, J.-B. Rodriguez, P. Christol, and I. Ribet-Mohamed, "Radiometric and noise characteristics of InAs-rich T2SL MWIR pin photodiodes," J. Eur. Opt. Soc.-Rapid Publ. 9, (2014).

13. O. Dier, C. Lin, M. Grau, and M.-C. Amann, "Selective and non-selective wet-chemical etchants for GaSb-based materials," Semicond. Sci. Technol. 19, 1250-1253 (2004). 
14. C. Huck, A. Toma, F. Neubrech, M. Chirumamilla, J. Vogt, F. De Angelis, and A. Pucci, "Gold Nanoantennas on a Pedestal for Plasmonic Enhancement in the Infrared," ACS Photonics 2, 497505 (2015).

15. P. T. Lin, V. Singh, Y. Cai, L. C. Kimerling, and A. Agarwal, "Air-clad silicon pedestal structures for broadband mid-infrared microphotonics," Opt. Lett. 38, 1031-1033 (2013).

16. H. W. Seo, L. W. Tu, Y. T. Lin, C. Y. Ho, Q. Y. Chen, L. Yuan, D. P. Norman, and N. J. Ho, "pGaN/InGaN/n-GaN pedestal nanorods: Effect of postgrowth annealing on the electrical performance," Appl. Phys. Lett. 94, 201907 (2009).

17. C. W. Wilmsen, ed., Physics and Chemistry of III-V Compound Semiconductor Interfaces (Springer US, 1985).

18. H. J. Bilz, G. Leonhardt, G. Kühn, K. Löschke, and A. Meisel, "ESCA-Untersuchungen an anodisch oxydierten $A_{\| I} B_{V}$-Verbindungen," Krist. Tech. 13, 363-368 (1978).

19. D. E. Aspnes, B. Schwartz, A. A. Studna, L. Derick, and L. A. Koszi, "Optical properties of anodically grown native oxides on some Ga-V compounds from 1.5 to $6.0 \mathrm{eV}$," J. Appl. Phys. 48, 3510 (1977).

20. G. P. Schwartz, "Analysis of native oxide films and oxide-substrate reactions on III-V semiconductors using thermochemical phase diagrams," Thin Solid Films 103, 3-16 (1983).

21. O. V. Sulima, A. W. Bett, and J. Wagner, "Anodic Oxidation of GaSb in Acid-Glycol-Water Electrolytes," J. Electrochem. Soc. 147, 1910-1914 (2000).

22. J. Mäkelä, M. Tuominen, M. Yasir, M. Kuzmin, J. Dahl, M. P. J. Punkkinen, P. Laukkanen, K. Kokko, and R. M. Wallace, "Oxidation of GaSb(100) and its control studied by scanning tunneling microscopy and spectroscopy," Appl. Phys. Lett. 107, 061601 (2015).

23. V. M. Bermudez, "First-principles study of the interaction of $\mathrm{H}_{2} \mathrm{O}$ with the $\mathrm{GaSb}(001)$ surface," J. Appl. Phys. 113, 184906 (2013).

24. D. Seo, J. Na, S. Lee, and S. Lim, "Behavior of a GaSb (100) Surface in the Presence of $\mathrm{H}_{2} \mathrm{O}_{2}$ in WetEtching Solutions," J. Phys. Chem. C 119, 24774-24780 (2015).

25. S. A. Jewett, J. A. Yoder, and A. Ivanisevic, "Surface modifications on InAs decrease indium and arsenic leaching under physiological conditions," Appl. Surf. Sci. 261, 842-850 (2012).

26. K. Tsunoda, Y. Matsukura, R. Suzuki, and M. Aoki, "Thermal instability of GaSb surface oxide," in B. F. Andresen, G. F. Fulop, C. M. Hanson, J. L. Miller, and P. R. Norton, eds. (2016), p. 98190S.

27. T. Taliercio, V. N. Guilengui, L. Cerutti, E. Tournié, and J.-J. Greffet, "Brewster "mode" in highly doped semiconductor layers: an all-optical technique to monitor doping concentration," Opt. Express 22, 24294 (2014).

28. M. J. Milla, F. Barho, F. González-Posada, L. Cerutti, M. Bomers, J.-B. Rodriguez, E. Tournié, and T. Taliercio, "Localized surface plasmon resonance frequency tuning in highly doped InAsSb/GaSb one-dimensional nanostructures," Nanotechnology 27, 425201 (2016).

29. S. Hayashi and T. Okamoto, "Plasmonics: visit the past to know the future," J. Phys. Appl. Phys. 45, 433001 (2012).

30. S. A. Maier, Plasmonics: Fundamentals and Applications (Springer, 2007).

31. L. Lin and Y. Yi, "Orthogonal and parallel lattice plasmon resonance in core-shell $\mathrm{SiO}_{2} / \mathrm{Au}$ nanocylinder arrays," Opt. Express 23, 130 (2015).

32. B. Luk'yanchuk, N. I. Zheludev, S. A. Maier, N. J. Halas, P. Nordlander, H. Giessen, and C. T. Chong, "The Fano resonance in plasmonic nanostructures and metamaterials," Nat. Mater. 9, 707-715 (2010).

33. A. E. Miroshnichenko, S. Flach, and Y. S. Kivshar, "Fano resonances in nanoscale structures," Rev. Mod. Phys. 82, 2257-2298 (2010).

34. T. Taliercio, V. Nt. Guilengui, L. Cerutti, J.-B. Rodriguez, F. Barho, M.-J. M. Rodrigo, F. GonzalezPosada, E. Tournié, M. Niehle, and A. Trampert, "Fano-like resonances sustained by Si doped InAsSb plasmonic resonators integrated in GaSb matrix," Opt. Express 23, 29423 (2015). 
35. M. Rei Vilar, J. El Beghdadi, F. Debontridder, R. Artzi, R. Naaman, A. M. Ferraria, and A. M. Botelho do Rego, "Characterization of wet-etched GaAs (100) surfaces," Surf. Interface Anal. 37, 673-682 (2005).

36. Lumerical Solutions Inc., http://www.lumerical.com/tcad-products/fdtd/.

37. S. Roux, P. Barritault, O. Lartigue, L. Cerutti, E. Tournié, B. Gérard, and A. Grisard, "Mid-infrared characterization of refractive indices and propagation losses in $\mathrm{GaSb} / \mathrm{Al}_{\mathrm{X}} \mathrm{Ga}_{1-\mathrm{x}} \mathrm{AsSb}$ waveguides," Appl. Phys. Lett. 107, 171901 (2015).

38. S. G. Kazarian and K. L. A. Chan, "ATR-FTIR spectroscopic imaging: recent advances and applications to biological systems," The Analyst 138, 1940 (2013). 